\title{
Laparoscopic TEP Inguinal Hernia Repair - Are We Overdoing It? A Comprehensive Study of 25 Cases of TEP Inguinal Hernia Repair Done Without the Use of Electrocautery and Without Any Mesh Fixation
}

\author{
Kaundinya Kiran Bharatam* \\ Specialist General Surgeon, Lifeline Hospital, Oman
}

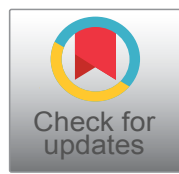

*Corresponding author: Dr. Kaundinya Kiran Bharatam, Specialist General Surgeon, Lifeline Hospital, Salalah, Oman

\begin{abstract}
Objectives: As one of the most effective and accepted minimally invasive techniques for inguinal hernia repair Laparoscopic Totally Extraperitoneal (TEP) repair is met with a learning curve and caution towards injury of major vessels/ nerves and mesh migration. Less experienced surgeon may tend to use excessive cautery and overzealous mesh fixation. Such practices in surgery can be avoided without any changes in immediate or late outcomes of the procedure.

Methods: Our case series of 25 patients who underwent the laparoscopic TEP inguinal hernia repair without use of cautery and without mesh fixation done over 2 years suggest the effcacy and outcomes of such practices allowing smooth and uneventful postoperative recovery and encouraging day care surgery.

Results: Assessment of patients for the outcomes of the said practice in laparoscopic TEP surgery included pain score assessment along with identification of early and late complications like Postoperative pain, seroma, infection, groin pain, and recurrence. Apart from a few patients having Postoperative pain responding to NSAIDs (non-steroidal anti-inflammatory drugs), none of the other complications were seen in the patient group.

Conclusion: Laparoscopic TEP for inguinal hernia repair does not need excessive cautery for plane dissection and also does not need mesh fixation necessarily. These practices have negligible outcome on hernia recurrence and enable smooth Postoperative recovery.
\end{abstract}

\section{Keywords}

Laparoscopic TEP inguinal hernia repair, Excessive electrocautery, Mesh fixation, Learning curves, Decreased postoperative pain, Day care hernia surgery

\section{Advances in Knowledge}

1. Laparoscopic Totally Extraperitoneal Repair for inguinal hernia is met with caution regarding damage to major vessels and nerves, especially in the triangle of pain and the triangle of doom and also for mesh migration.

2. Less experienced surgeons may tend to be a bit overzealous in their use of electrocautery and mesh fixation.

3. We proposed minimal or no use of electrocautery and non-fixation of mesh for our study.

4. The study is focused on highlighting easier and faster practices in the procedure to encourage laparoscopic TEP for hernia repair as day care surgery for hernia even by less experienced surgeons.

\section{Application to Patient Care}

1. By using no or minimal electrocautery in dissection of planes in the preperitoneum and depending on pneumodissection or telescope dissection, the visualization was optimal without tissue charring, smoke or any unwarranted bleeding.

2. Postoperative pain especially immediate secondary to thermal injury or secondary inflammation response to the cautery was least.

3. A large mesh was used $(15 \times 12.5 \mathrm{~cm})$ and this did not require fixation as it occupied the entire preperitoneal space without scope for migration or slippage.

Citation: Bharatam KK (2018) Laparoscopic TEP Inguinal Hernia Repair - Are We Overdoing It? A Comprehensive Study of 25 Cases of TEP Inguinal Hernia Repair Done Without the Use of Electrocautery and Without Any Mesh Fixation. Clin Med Rev Case Rep 5:232. doi.org/10.23937/2378-3656/1410232 Accepted: August 15, 2018: Published: August 17, 2018

Copyright: (c) 2018 Bharatam KK. This is an open-access article distributed under the terms of the Creative Commons Attribution License, which permits unrestricted use, distribution, and reproduction in any medium, provided the original author and source are credited. 
4. Such practices decreased operative time and instilled confidence in performing surgery in day care setting.

\section{Introduction}

Laparoscopic Totally Extraperitoneal Repair of inguinal hernia is a popular and prevalent technique for laparoscopic inguinal hernia repair. It has several advantages apart from being a minimally invasive procedure like the possibility of bilateral hernia repair (if present) and also the repair of femoral hernia component simultaneously (if present). The technique is not without a learning curve and requires a surgeon to understand the anatomy of the posterior inguinal canal along with the knowledge of dangerous areas like the triangle of doom and triangle of pain responsible for catastrophic consequences like major vessel bleeding and nerve injury. The usual expected complications are postoperative pain, seroma formation, chronic groin pain, scrotal swelling, and mesh infection. To avoid these complications a surgeon usually treads in the region of preperitoneum with caution and may be led to use excessive use of electrocautery and overzealous mesh fixation. Such practices may provide a good vision for surgery and allow no scope for mesh migration, still they allow the possibility for increased Postoperative pain, tissue damage, nerve injury, and increased operative times.

From a period of July 2016 till May 2018 (2 years), 25 inguinal hernia repairs were done by the laparoscopic TEP inguinal hernia repair technique. An approach was chosen to avoid the use of electrocautery completely and to avoid any mesh fixation device intraoperatively. For this purpose, the use of electrocautery was kept standby only for uncontrollable bleeding (if any) and a larger size of the mesh was chosen $(15 \times 12.5 \mathrm{~cm}$ Ethicon prolene hernia mesh) for all such cases. The primary goal was to ensure smooth postoperative recovery without pain, allow for single day discharge of such patients, and even minimize postoperative complications like seroma, infection, and ultimately recurrence.

\section{Methods}

The study was performed in Lifeline hospitals, Salalah, Oman - a private hospital. Ethical permission was granted by the medical director of the hospital and the study was performed with the full information of the medical director and with informed consent from the patient. For the said period of study, the cases selected were only Uncomplicated Unilateral/Bilateral inguinal hernias - Nyhus classification until type 3A. All the patients were ASA 1-2 fitness for surgery and were fully informed about the procedure and the technique used. Patients with complicated and/or recurrent hernias were not selected for surgery and underwent inguinal hernia repair by the Lichtenstein's open approach or laparoscopic repair (TEP/TAPP) as per need. The patients once diagnosed were asked to undergo pre-anesthesia evaluation. Once fitness for surgery was con- firmed, the patient was asked to come to the hospital 2 hours prior to surgery on an empty stomach since the previous night meal. The surgery was done within 3 hours of admission. The choice of procedure was Laparoscopic Totally Extraperitoneal Inguinal Hernia Repair under general anesthesia and the approximate duration of surgery was between $60-90$ minutes. The patient was placed in supine position with legs apart. Access to the preperitoneal space was obtained by port placement anterior to the posterior rectus sheath. Once the access was confirmed by the 'angel hair' appearance, dissection of preperitoneal space was initiated. The pneumo pressure was kept around $1012 \mathrm{mmHg}$. Using blunt dissection anterior to the transversalis fascia and the peritoneum, pneumodissection was used to create space in the preperitoneal space along with telescopic dissection. Once enough space was created to visualize the pubic symphysis medially, the cord structures entering the deep ring laterally, and adequate lateralization till Anterior Superior Iliac Spine, the hernia was addressed. For indirect hernias, cord dissection was done to isolate the sac completely which was closed with an endoloop. Direct hernia defects were identified and contents in the hernia defect were reduced. Once hernia was completely reduced, a large $15 \times 12.5 \mathrm{~cm}$ Ethicon prolene hernia mesh was placed in situ and adjusted to completely to cover the defect. Overlaps and gaps were carefully removed to avoid seroma formation. In bilateral hernias similar mesh was placed bilaterally. No fixation device was used as the mesh size completely occupied the dissected preperitoneal space and there was no scope of mesh migration or slippage. Procedure was completed after reducing the pneumo completely and closing the port sites with appropriate sutures. Postoperatively, patients were given single dose antibiotics and analgesia was kept on required basis only. Early ambulation and scrotal support was encouraged. An objective pain score assessment was done and patients were discharged on the next day of the procedure. The patients were advised to undergo regular daily activities except for lifting heavy weights or involving in strenuous activity/exercise. They were reviewed on Postoperative day 7 for suture removal. A second Postoperative visit was planned on Postoperative day 15 for any complications/ complaints.

\section{Results}

In the 25 cases operated from July 2016 till may, 2018 in the same centre with the same surgeon, assistant and anesthesiologist, the average age of patients was found to be around 30 (15-45 yrs). They were predominantly males with only 2 female patients with inguinal hernia. The body mass index varied from 25-32 although it was not a criteria for inclusion/exclusion for the study. The average stay of the patient in the hospital was around 26 hours ( 24 - 30 hours). Most patients had a pain score of less than 4 and only 2 patients had a pain score of 4-8. These patients were given NSAIDs (non-steroidal 
Table 1: Postoperative pain score for patients in the study.

\begin{tabular}{|l|l|}
\hline Pain score & No. of Patients \\
\hline Score $1-4$ & 23 \\
\hline Score $4-8$ & 2 \\
\hline Score $>8$ & 0 \\
\hline
\end{tabular}

Table 2: Postoperative complications expected in the patients in study.

\begin{tabular}{|l|l|}
\hline Complication & No. of Patients \\
\hline Postoperative Pain (Score > 8) & 0 \\
\hline Postoperative Seroma/Scrotal Collection & 0 \\
\hline Postoperative Scrotal Swelling & 0 \\
\hline Pain Groin Region & 0 \\
\hline Chronic Groin Pain & 0 \\
\hline Wound/Mesh Infection - Immediate & 0 \\
\hline Wound/Mesh Infection - Delayed & 0 \\
\hline Evidence of Bleeding - Immediate & 0 \\
\hline Evidence of Bleeding - Delayed & 0 \\
\hline Recurrence of Hernia Symptoms - Immediate & 0 \\
\hline Recurrence of Hernia Symptoms - Delayed & 0 \\
\hline Wound Gaping/Dehiscence & 0 \\
\hline
\end{tabular}

anti inflammatory drugs) and they responded well. They were willing for discharge on next day of surgery as all patients in the study. Follow up of the patients was requested on $7^{\text {th }}$ day of surgery for stitch removal and on $15^{\text {th }}$ Postoperative day for regular follow up and for any complaints/complications. All patients had no complaints/complications during both the Postoperative visits (Table 1).

\section{Discussion}

Performing laparoscopic inguinal hernia repair by the totally extraperitoneal approach requires an understanding of the posterior inguinal canal anatomy. This may even necessitate performing the Stoppa open hernia repair prior for better clarity [1]. Knowledge of possible injury to major vessels and nerves is highlighted by the presence of the triangle of doom and pain respectively. Those proceeding ahead in their learning curves of the TEP repair usually proceed with caution with regards to mainly the said factors [2]. In the absence of proper mentorship and guidance by an experienced laparoscopic surgeon to learn the procedure, many a times the surgeon resorts to excessive usage of electrocautery, trying to avoid any possible bleeding, even in avascular planes. This is mainly due to fear of loss of vision and risk of conversion to open technique. Tissue charring, damage to fine nerves, increased inflammatory responses, transmitted energy damage can result in local seroma formation, increased Postoperative pain, possible chronic groin pain and rarely transmitted energy damage to intra and extraperitoneal structures $[3,4]$. Excessive electrocautery usage produces a lot of smoke which also hampers vision. The charring of tissue further hampers the plane dissection and encourages the surgeon to go in wrong planes. This causes more bleeding/oozing and the repeat use of electrocautery initiating a vicious cycle. The final outcome is prolonged oper- ative times along with the previously said postoperative problems $[5,6]$. Once the cord structures are identified and hernia defect is reduced, a mesh placement is again met with similar anxiety to prevent mesh migration and thus the recurrence of the hernia repaired [7]. Though initially anatomic mesh limited to the size of the defect were advocated, these required fixation to the pubic tubercle and the conjoint tendon (Table 2).

Presently larger mesh placement is accepted without any necessary fixation $[8,9]$. Although usage of fixation devices like tackers or glue have been popular they are also met with increased Postoperative pain and sometimes even tissue reactions causing local and scrotal collections [10]. Use of fixation devices like the tackers in the conjoint tendon or the pubic tubercle are mainly causative factors for Postoperative pain and discourage early ambulation. This may again promote seroma formation and also increase hospital stay duration. The main reason for using tackers is to avoid mesh migration and ultimately recurrence of hernia repaired especially when small mesh sizes are preferred. Our study was mainly focused on relieving this anxiety and easing the procedure of dissection for reaching the required structures extraperitoneally for hernia repair in the TEP approach. Pneumo is excellent in dissection and allows easy separation and visualization of the planes. Also the pressure is capable of allowing hemostasis in fine capillaries or similar vessels. Traction and counter traction used in dissection of planes usually is maximally effective in maximal dissection even till the point of maximum lateralization till the ASIS and only direct instrument damage to major vessels like the inferior epigastric arteries and the iliac veins need be avoided. Our study also focused on increasing surgical efficacy and encouraging day care surgeries for inguinal hernia repair. Prospective studies do not suggest a difference in the incidence of recurrence rates with or without any fixation device usage $[10,11]$. At the same time they also suggest increased Postoperative pain and tissue reactions in the presence of such fixations used [12]. Thus if mesh fixation can be avoided by usage of larger meshes to avoid any possible migration, then both the problems of recurrence and Postoperative pain can be addressed at the same time $[5,8,9,11]$. Inguinal hernias are being considered effectively treated by the TEP laparoscopic approach allowing even bilateral repairs in the same sitting by minimally invasive technique. Even Though a thorough knowledge of the anatomy and the possible complications is mandatory, the usage of excessive force in form of excessive electrocautery and overzealous fixation can be avoided and this has its many advantages [5]. The proper usage of pneumo dissection and usage of large mesh can very well make surgery simpler, and decrease the metabolic response to the surgical trauma. The patient can be encouraged to ambulate early and the decreased postoperative pain would allow the patient to choose day care approach and even allow faster recovery to regular activity and to work [11]. 


\section{Conclusion}

Laparoscopic TEP repair for inguinal hernia is very effective for both direct and indirect inguinal hernias. Instilling confidence by knowledge of surgery, the anatomy of the inguinal canal (laparoscopic), and the knowledge of the complications should be supplanted by the assurance that excessive electrocautery and mesh fixation is unnecessary for smooth and uneventful postoperative outcomes and for prevention of any form of hernia recurrence. This will in turn decrease the learning curve period and encourage day care surgery for a majority of uncomplicated inguinal hernias $[2,5]$.

\section{References}

1. Lal P, Kajla RK, Chander J, Ramteke VK (2004) Laparoscopic total extraperitoneal (TEP) inguinal hernia repair: Overcoming the learning curve. Surg Endosc 18: 642-655.

2. Bansal VK, Krishna A, Misra MC, Kumar S (2016) Learning curve in laparoscopic inguinal hernia repair: Experience at a tertiary care centre. Indian J Surg 78: 197-202.

3. Kojima S, Sakamoto T, Honda M, Nishiguchi R, Ogawa F (2016) Rare complication after totally extraperitoneal endoscopic inguinal hernia repair: Small bowel perforation without peritoneal disruption. Asian J Endosc Surg 9: 311-313.

4. Broek RP, Wilbers J, van Goor H (2011) Electrocautery causes more ischemic peritoneal tissue damage than ultrasonic dissection. Surg Endosc 25: 1827-1834.

5. Ferzli G, Sayad P, Huie F, Hallak A, Usal H (1998) Endo- scopic extraperitoneal herniorrhaphy. A 5year experience. Surg Endosc 12: 1311-1313.

6. Kumar S, Joshi M, Chaudhary S (2009) 'Dissectalgia' following TEP, a new entity: its recognition and treatment. Results of a prospective randomized controlled trial. Hernia 13: $591-596$

7. Choy C, Shapiro K, Patel S, Graham A, Ferzli G (2004) Investigating a possible cause of mesh migration during totally extraperitoneal (TEP) repair. Surg Endosc 18: 523-525.

8. Mayer F, Niebuhr H, Lechner M, Dinnewitzer A, Köhler G, et al. (2016) When is mesh fixation in TAPP-repair of primary inguinal hernia repair necessary? The register-based analysis of 11,230 cases. Surg Endosc 30: 4363-4371.

9. Claus CM, Rocha GM, Campos AC, Bonin EA, Dimbarre D, et al. (2016) Prospective, randomized and controlled study of mesh displacement after laparoscopic inguinal repair: Fixation versus no fixation of mesh. Surg Endosc 30: 11341140.

10. Horisberger K, Jung MK, Zingg U, Schöb O (2013) Influence of type of mesh fixation in endoscopic totally extraperitoneal hernia repair (TEP) on long-term quality of life. World J Surg 37: 1249-1257.

11. Teng YJ, Pan SM, Liu YL, Yang KH, Zhang YC, et al. (2011) A meta-analysis of randomized controlled trials of fixation versus nonfixation of mesh in laparoscopic total extraperitoneal inguinal hernia repair. Surg Endosc 25: 2849-2858.

12. Gutlic N, Rogmark P, Nordin P, Petersson U, Montgomery A (2016) Impact of mesh fixation on chronic pain in Total Extraperitoneal Inguinal Hernia Repair (TEP): A nationwide register based Study. Ann Surg 263: 1199-1206. 